Rapid Reviews COVID-19

\title{
Review 3: "Neutralization of N501Y mutant SARS- CoV-2 by BNT162b2 vaccine-elicited sera"
}

\section{Geraldo Passos ${ }^{1}$}

${ }^{1}$ Associate Professor, University of Sao Paulo School of Dentistry of Ribeirao Preto, Brazil

Published on: Feb 07, 2021

License: Creative Commons Attribution 4.0 International License (CC-BY 4.0). 


\section{$\underline{\text { RR:C19 Evidence Scale rating by reviewer: }}$}

- Reliable. The main study claims are generally justified by its methods and data. The results and conclusions are likely to be similar to the hypothetical ideal study. There are some minor caveats or limitations, but they would/do not change the major claims of the study. The study provides sufficient strength of evidence on its own that its main claims should be considered actionable, with some room for future revision.

\section{Review:}

The authors of the manuscript "Neutralization of N501Y mutant SARS-CoV-2 by BNT162b2 vaccineelicited sera" tested the effect of blood serum from 20 people immunized with the BNT162b2 mRNA vaccine that encodes the Spike protein from Sars-Cov-2. They found that the serum from these immunized people reduced the number of lysis plaques in an in vitro Sars-Cov-2 infection experiment. They tested the original strain of this virus (N501) and an artificially generated virus mutant lineage harboring the replacement mutation (N501Y). As reported by the authors, a limitation of this experiment is that the artificially created strain only harbors the N501Y mutation and not the other mutations found in the new variants from the UK or South Africa. It is objective and straightforward communication, but the results suggest that the BNT162b2 mRNA vaccine could protect against the highly contagious Sars-Cov-2 N501Y variant. New variants of Sars-Cov-2 are expected because the virus mutates in its genome. RNA viruses such as Sars-Cov2 do not have a repair system and continuously present new mutations. The question is how these new Sars-Cov2 variants survive and spread. The critical point is the spread of the virus facilitated by person-to-person contact. This way, Sars-Cov-2 reproduces more quickly, and new mutations are emerging. The new variant of Sars-Cov-2, which has the N501Y mutation in the Spike protein, is worrying because it is highly infectious.

However, we still do not know if this new variant can cause a more severe COVID-19. We need to know if the versions of the vaccines against COVID-19 already available and administered worldwide protect against this new variant. The results of this work, from in vitro experiments, indicate that the blood serum of people who received the BNT162b2 mRNA vaccine contains neutralizing antibodies against an artificial strain of Sars-Cov-2 that has the N501Y mutation. 